\title{
Integer Programming Duality
}

\author{
M. Guzelsoy* $\quad$ T. K. Ralphs ${ }^{\dagger}$
}

July, 2010

\section{Introduction}

This article describes what is known about duality for integer programs. It is perhaps surprising that many of the results familiar from linear programming (LP) duality do extend to integer programming. However, this generalization requires adoption of a more general point of view on duality than is apparent from studying the linear programming case. Duality theory, as we shall define it here, is generally concerned with the development of methods for determining the effect on the optimal solution value of perturbations in the right-hand side of a mathematical program, which can be thought of as specifying the resources available to operate a system. The study of duality is hence the study of a parameterized family of mathematical programming instances and is closely related to parametric programming and sensitivity analysis. More formally, duality theory can be thought of as the study of the so-called value function, which is a function that takes a right-hand side vector as input and returns the optimal solution value of one of a family of integer programming instances parameterized by that right-hand side vector. In the linear programming case, the value function is piecewise linear and convex (assuming minimization), which is the reason for the tractability of the linear programming dual problem. In the integer programming case, the value function has a more complex structure, as we shall see.

In what follows, we consider a mixed integer linear program (MILP) (referred to as the primal problem) of the form

$$
z_{I P}=\min _{x \in \mathcal{S}} c x
$$

where $c \in \mathbb{R}^{n}$ is the objective function vector and the feasible region

$$
\mathcal{S}=\left\{x \in \mathbb{Z}_{+}^{r} \times \mathbb{R}_{+}^{n-r} \mid A x=b\right\}
$$

is defined by a constraint matrix $A \in \mathbb{Q}^{m \times n}$ and a right-hand side vector $b \in \mathbb{R}^{m}$. Note that for simplicity of notation, we omit the transpose symbol for the inner product of vectors and for matrix-vector products. By convention, $z_{I P}=\infty$ if $\mathcal{S}=\emptyset$ and $z_{I P}=-\infty$ if for every $x \in \mathcal{S}$, there exists $y \in \mathcal{S}$ such that $c y<c x$.

${ }^{*}$ School of Industrial and Systems Engineering, Georgia Institute of Technology, Atlanta, GA 30332, mguzelsoy@gatech.edu

${ }^{\dagger}$ Department of Industrial and Systems Engineering, Lehigh University, Bethlehem, PA 18015, ted@lehigh.edu 
The goal is to determine $z_{I P}$ and an optimal solution $x^{*}$ such that $c x^{*}=z_{I P}$ (if $z_{I P}$ is finite), where $c x$ is the solution value of $x \in \mathcal{S}$. The variables indexed by the set $I=\{1, \ldots, r\} \subseteq N=\{1, \ldots, n\}$ (if $r>0$, otherwise $I=\emptyset$ ) are the integer variables and the remaining variables, indexed by the set $C=N \backslash I$, are the continuous variables. The LP obtained from a given MILP by removing the integrality requirements on the variables, i.e., setting $r=0$, is referred to as the associated $L P$ relaxation. Similarly, the associated pure integer linear program (PILP) is obtained by requiring all variables to be integer, i.e., setting $r=n$.

By parameterizing (1), we obtain the value function $z: \mathbb{R}^{m} \rightarrow \mathbb{R} \cup\{ \pm \infty\}$ defined as

$$
z(d)=\min _{x \in \mathcal{S}(d)} c x
$$

where, for a given $d \in \mathbb{R}^{m}, \mathcal{S}(d)=\left\{x \in \mathbb{Z}_{+}^{r} \times \mathbb{R}_{+}^{n-r} \mid A x=d\right\}$. A dual function $F: \mathbb{R}^{m} \rightarrow \mathbb{R} \cup\{ \pm \infty\}$ is a function that bounds the value function over the set $\mathbb{R}^{m}$, that is,

$$
F(d) \leq z(d) \quad \forall d \in \mathbb{R}^{m}
$$

Dual functions and methods for constructing them are the central focus of duality theory. The aim in constructing a dual function $F$ is for it to closely approximate the value function $z$. In particular, we usually want a dual function whose value at $b$ is as close as possible to $z(b)$. The most general form of the dual problem is then

$$
z_{D}=\max \left\{F(b): F(d) \leq z(d), d \in \mathbb{R}^{m}, F: \mathbb{R}^{m} \rightarrow \mathbb{R}\right\},
$$

which is an optimization problem over the space of dual functions. We call $F$ a weak dual function if it is feasible for (5), i.e., it satisfies (4), and a strong dual function with respect to $b$ if it is optimal for (5), i.e., satisfies $F(b)=z(b)$. Note that we allow only real-valued functions, so that (5) is infeasible if $z_{I P}=-\infty$

and $z_{D} \leq z_{I P}$ otherwise. If $z_{I P}$ is finite, the value function itself (or a real-valued extension of it) is a strong dual function, i.e., $z_{D}=z_{I P}$.

Example 1 Consider the following MILP instance with a fixed right-hand side $b$

$$
\begin{aligned}
\min & 3 x_{1}+\frac{7}{2} x_{2}+3 x_{3}+6 x_{4}+7 x_{5} \\
\text { s.t } & 6 x_{1}+5 x_{2}-4 x_{3}+2 x_{4}-7 x_{5}=b \text { and } \\
& x_{1}, x_{2}, x_{3} \in \mathbb{Z}_{+}, x_{4}, x_{5} \in \mathbb{R}_{+} .
\end{aligned}
$$

The value function $z$ of this sample problem together with dual functions $F_{1}$ and $F_{2}$ are given in Figure 1. Note that the value function is better approximated by $F_{1}$ for right-hand side values $b>0$ and by $F_{2}$ otherwise. In addition, observe that the dual functions are strong for a subset of right-hand side values and weak otherwise.

\section{Properties of the Value Function}

The study of duality begins with the study of the value function itself. In particular, we want to know what the structure of the value function is and to what class of functions it may belong. Our fist observation is 


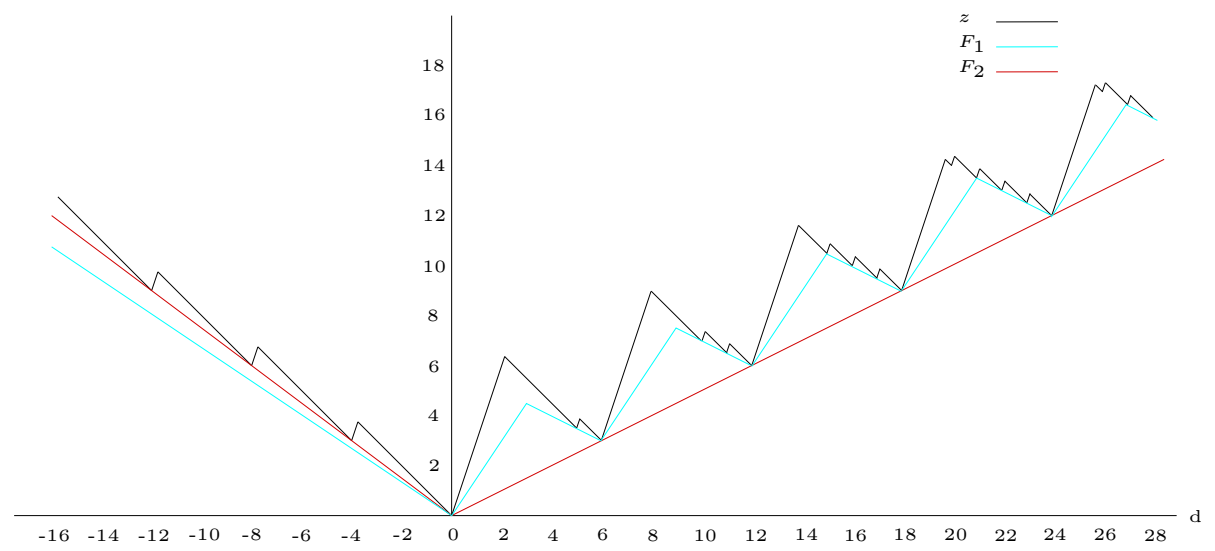

Figure 1: The value function and sample dual functions for (6).

that the value function is subadditive. A function $F$ is subadditive over a domain $\Theta$ if $F\left(\lambda_{1}\right)+F\left(\lambda_{2}\right) \geq$ $F\left(\lambda_{1}+\lambda_{2}\right)$ for all $\lambda_{1}, \lambda_{2}, \lambda_{1}+\lambda_{2} \in \Theta$. The value function itself is subadditive over the domain $\Omega=\{d \in$ $\left.\mathbb{R}^{m} \mid S(d) \neq \emptyset\right\}$. To see why, let $d_{1}, d_{2} \in \Omega$ and suppose $z\left(d_{i}\right)=c x_{i}$ for some $x_{i} \in \mathcal{S}\left(d_{i}\right), i=1,2$. Then, $x_{1}+x_{2} \in \mathcal{S}\left(d_{1}+d_{2}\right)$ and hence $z\left(d_{1}\right)+z\left(d_{2}\right)=c\left(x_{1}+x_{2}\right) \geq z\left(d_{1}+d_{2}\right)$. Subadditivity reflects the intuitively natural property of increasing returns to scale that we expect the value function to have.

For PILPs, Blair and Jeroslow [1982] showed that a procedure similar to Gomory's cutting plane procedure can be used to construct the value function in a finite number of steps, although the representation from this procedure may have exponential size. They were thus able to characterize the class of functions to which value functions belong, namely, Gomory functions, a subset of a more general class called Chvátal functions.

Definition 1 Let $\mathcal{L}^{m}=\left\{f: \mathbb{R}^{m} \rightarrow \mathbb{R} \mid f\right.$ is linear $\}$. Chvátal functions are the smallest set of functions $\mathscr{C}$ such that

1. If $h \in \mathcal{L}^{m}$, then $h \in \mathscr{C}$.

2. If $h_{1}, h_{2} \in \mathscr{C}$ and $\alpha, \beta \in \mathbb{Q}_{+}$, then $\alpha h_{1}+\beta h_{2} \in \mathscr{C}$.

3. If $h \in \mathscr{C}$, then $\lceil h\rceil \in \mathscr{C}$.

Gomory functions are the smallest set of functions $\mathscr{G} \subseteq \mathscr{C}$ with the additional property that

4. If $h_{1}, h_{2} \in \mathscr{G}$, then $\max \left\{h_{1}, h_{2}\right\} \in \mathscr{G}$.

Note that both Chvátal and Gomory functions are subadditive and every Gomory function can be written as the maximum of finitely many Chvátal functions. Blair and Jeroslow [1982] show for a PILP in the form (1) with $z(0)=0$ that (i) the value function over the domain $\Omega$ is a Gomory function and (ii) there always exists an optimal solution to the dual problem (5) that is a Chvátal function.

For MILPs, Blair and Jeroslow [1984] argue that the value function can still be represented by a Gomory function if $c_{j}=0 \forall j \in C$ or can be written as a minimum of finitely many Gomory functions. A deeper 
result is contained in the subsequent work of Blair [1995], who showed that the value function of an MILP can be written as a Jeroslow formula, consisting of a Gomory function and a correction term. For a given $d \in \Omega$, let the set $\mathscr{E}$ consist of the index sets of dual feasible bases of the linear program

$$
\min \left\{c_{C} x_{C}: A_{C} x_{C}=d, x \geq 0\right\}
$$

By the rationality of $A$, we can choose $M \in \mathbb{Z}_{+}$such that $M A_{E}^{-1} a^{j} \in \mathbb{Z}^{m}$ for all $E \in \mathscr{E}, j \in I$, where $a^{j}$ is the $j^{\text {th }}$ column of $A$. For $E \in \mathcal{E}$, let $v_{E}$ be the corresponding basic feasible solution to the dual of

$$
\min \left\{\frac{1}{M} c_{C} x_{C}: \frac{1}{M} A_{C} x_{C}=d, x \geq 0\right\},
$$

which is a scaled version of (7). Finally, for a right-hand side $d$ and $E \in \mathcal{E}$, let $\lfloor d\rfloor_{E}=A_{E}\left\lfloor A_{E}^{-1} d\right\rfloor$.

Theorem 2 (Blair [1995]) For the MILP (1), there is a $g \in \mathscr{G}$ such that

$$
z(d)=\min _{E \in \mathscr{E}} g\left(\lfloor d\rfloor_{E}\right)+v_{E}\left(d-\lfloor d\rfloor_{E}\right)
$$

for all $d \in \Omega$.

\section{The Subadditive Dual}

The dual (5) is rather general, so a natural question is whether it is possible to restrict the space of functions in order to obtain a tractable method for constructing a strong dual function. Note that

$$
\begin{aligned}
F(d) \leq z(d), d \in \mathbb{R}^{m} & \Longleftrightarrow F(d) \leq c x, x \in \mathcal{S}(d), d \in \mathbb{R}^{m} \\
& \Longleftrightarrow F(A x) \leq c x, x \in \mathbb{Z}_{+}^{r} \times \mathbb{R}_{+}^{n-r} .
\end{aligned}
$$

Restricting $F$ to be linear, (5) reduces to $\max \left\{v b \mid v A \leq c, v \in \mathbb{R}^{m}\right\}$ by (10), which is the dual of the LP relaxation of the original MILP. Jeroslow [1979] showed that restricting the search space to only convex functions also results in the same optimal solution value obtained in the linear case. Therefore, these restrictions result in a dual that is no longer guaranteed to produce a strong dual function.

Johnson [1973, 1974, 1979] and later Jeroslow [1978] developed the idea of restricting the search space to a certain subset of subadditive functions. It is clear that this restriction retains the desirable property of strong duality, since the value function is itself subadditive over $\Omega$.

From (10), if we set $\Gamma^{m} \equiv\left\{F: \mathbb{R}^{m} \rightarrow \mathbb{R} \mid F(0)=0, F\right.$ is subadditive $\}$, then we can rewrite (5) as the subadditive dual

$$
\begin{aligned}
z_{D}=\max & F(b) \\
& F\left(a^{j}\right) \leq c_{j} \quad \forall j \in I, \\
& F\left(a^{j}\right) \leq c_{j} \quad \forall j \in C, \text { and } \\
& F \in \Gamma^{m},
\end{aligned}
$$


where the function $\bar{F}$ is the upper d-directional derivative of $F$ at zero and is defined by

$$
\bar{F}(d)=\limsup _{\delta \rightarrow 0^{+}} \frac{F(\delta d)}{\delta} \forall d \in \mathbb{R}^{m} .
$$

Intuitively, the role of $\bar{F}$ in (11) is to ensure that solutions to (11) have gradients that do not exceed those of the value function near zero, since the subadditivity of $F$ alone is not enough to ensure this in the case of MILP.

The main results concerning the relationship between the primal and dual problems in LP duality can be generalized to the MILP case.

Theorem 3 For the primal problem (1) and its subadditive dual (11), the following statements hold:

1. (Weak Duality by Jeroslow [1978, 1979]) Let $x$ be a feasible solution to the primal problem and let $F$ be a feasible solution to the subadditive dual. Then, $F(b) \leq c x$.

2. If the primal problem (resp., the dual) is unbounded then the dual problem (resp., the primal) is infeasible.

3. If the primal problem (resp., the dual) is infeasible, then the dual problem (resp., the primal) is infeasible or unbounded.

4. (Strong duality by Jeroslow [1978, 1979], Wolsey [1981]) If the primal problem (resp., the dual) has a finite optimum, then so does the dual problem (resp., the primal) and they are equal.

The proof of weak duality and the associated results follow directly from the construction of dual problem. However, in order to show strong duality holds for the case where $\Omega \subset \mathbb{R}^{m}$, we need an additional result of Blair and Jeroslow [1977], which states that any given MILP can be "extended" to one that is feasible for all right-hand sides and whose value function agrees with that of the original MILP for all right-hand sides $d \in \Omega$.

Example 2 For the MILP (6), the subadditive dual problem is

$$
\begin{aligned}
\max F(b) & \\
F(6) & \leq 3 \\
F(5) & \leq \frac{7}{2} \\
F(-4) & \leq 3 \\
\bar{F}(2) & \leq 6 \\
\bar{F}(-7) & \leq 7 \\
F & \in \Gamma^{1} .
\end{aligned}
$$

Note from Figure 1 that $F_{2}(d)=\max \left\{\frac{d}{2},-\frac{3 d}{4}\right\} \forall d \in \mathbb{R}$ is feasible to dual problem and furthermore, optimal for the right-hand side values $b \in\{\ldots,-8,-4,0,6,12, \ldots\}$. 
The subadditive dual (11) can also be used to extend familiar concepts such as reduced costs and the complementary slackness conditions to MILPs. For a given optimal solution $F^{*}$ to (11), the reduced costs can be defined as $c_{j}-F^{*}\left(a^{j}\right)$ for $j \in I$ and $c_{j}-\bar{F}^{*}\left(a^{j}\right)$ for $j \in C$. These reduced costs have an interpretation similar to that in the LP case, except that we do not have the same concept of "sensitivity ranges" within which the computed bounds are exact. On the other hand, complementary slackness conditions, if satisfied, yield a certificate of optimality for a given primal-dual pair of feasible solutions and can be stated as follows.

Theorem 4 (Jeroslow [1978], Johnson [1979], Bachem and Schrader [1980], Wolsey [1981]) For a given right-hand side b, let $x^{*}$ and $F^{*}$ be feasible solutions to to the primal problem (1) and the subadditive dual problem (11). Then, $x^{*}$ and $F^{*}$ are optimal if and only if

$$
\begin{aligned}
x_{j}^{*}\left(c_{j}-F^{*}\left(a^{j}\right)\right) & =0, \forall j \in I, \\
x_{j}^{*}\left(c_{j}-\bar{F}^{*}\left(a^{j}\right)\right) & =0, \forall j \in C, \text { and } \\
F^{*}(b) & =\sum_{j \in I} F^{*}\left(a^{j}\right) x_{j}^{*}+\sum_{j \in C} \bar{F}^{*}\left(a^{j}\right) x_{j}^{*} .
\end{aligned}
$$

The subadditive duality framework also allows the use of subadditive functions to obtain inequalities valid for the convex hull of the feasible region. It is easy to see that for any $d \in \Omega$ and $F \in \Gamma^{m}$ with $\bar{F}\left(a^{j}\right)<$ $\infty \forall j \in C$, the inequality

$$
\sum_{j \in I} F\left(a^{j}\right) x_{j}+\sum_{j \in C} \bar{F}\left(a^{j}\right) x_{j} \geq F(d)
$$

is satisfied for all $x \in \mathcal{S}(d)$. Using this property, Johnson [1973] and later Jeroslow [1978] showed that any valid inequality for $\mathcal{S}(d)$ is either equivalent to or dominated by an inequality in the form of (15).

As an extension to this result, Bachem and Schrader [1980] showed that the convex hull of $\mathcal{S}(d)$ can be represented using only subadditive functions and that rationality of $A$ is enough to ensure the existence of such a representation, even if the convex hull is unbounded. For a fixed right-hand side, it is clear that only finitely many subadditive functions are needed to obtain a complete description, since every rational polyhedron has finitely many facets. In addition, Wolsey [1979] showed that for PILPs, there exists a finite representation that is valid for all right-hand sides.

Theorem 5 (Wolsey [1979]) For a PILP in the form (1), there exist finitely many subadditive functions $F_{i}, i=1, \ldots, k$, such that

$$
\operatorname{conv}(\mathcal{S}(d))=\left\{x: \sum_{j=1}^{n} F_{i}\left(a^{j}\right) x_{j} \geq F_{i}(d), i=1, \ldots, k, x \geq 0\right\}
$$

for any $d \in \Omega$.

\section{Constructing Dual Functions}

Dual functions can be grouped into three categories: (1) those obtained from known families of relaxations, (2) those obtained as a by-product of a primal solution algorithm, such as branch-and-bound, and (3) those 
constructed explicitly in closed form using a finite procedure.

Cutting Plane Method. Cutting plane algorithms are a broad class of methods for obtaining lower bounds on the optimal solution value of a given MILP by iteratively generating inequalities (called cutting planes or cuts) valid for the convex hull of $\mathcal{S}$. In iteration $k$, the algorithm solves the following linear program:

$$
\begin{aligned}
\min & c x \\
\text { s.t. } & A x=b \\
& \Pi x \geq \Pi_{0} \\
& x \geq 0,
\end{aligned}
$$

where $\Pi \in \mathbb{R}^{k \times n}$ and $\Pi_{0} \in \mathbb{R}^{k}$ represents the cutting planes generated so far. The LP dual of (17), i.e.,

$$
\begin{array}{cc}
\max & v b+w \Pi_{0} \\
& v A+w \Pi \leq c \\
v & \in \mathbb{R}^{m}, w \in \mathbb{R}_{+}^{k},
\end{array}
$$

is also a dual problem for the original primal MILP. Assuming that a subadditive representation (15) of each cut is known, the $i^{t h}$ cut can be expressed parametrically as a function of the right-hand side $d \in \mathbb{R}^{m}$ in the form

$$
\sum_{j \in I} F_{i}\left(\sigma_{i}\left(a^{j}\right)\right) x_{j}+\sum_{j \in C} \bar{F}_{i}\left(\bar{\sigma}_{i}\left(a^{j}\right)\right) x_{j} \geq F_{i}\left(\sigma_{i}(d)\right)
$$

where $F_{i}$ is the subadditive function representing the cut, and the functions $\sigma_{i}, \bar{\sigma}_{i}: \mathbb{R}^{m} \rightarrow \mathbb{R}^{m+i-1}$ are defined by

- $\sigma_{1}(d)=\bar{\sigma}_{1}(d)=d$,

- $\sigma_{i}(d)=\left[\begin{array}{llll}d & F_{1}\left(\sigma_{1}(d)\right) & \ldots & F_{i-1}\left(\sigma_{i-1}(d)\right)\end{array}\right]$ for $i \geq 2$, and

- $\bar{\sigma}_{i}(d)=\left[\begin{array}{llll}d \bar{F}_{1}\left(\bar{\sigma}_{1}(d)\right) & \ldots & \bar{F}_{i-1}\left(\bar{\sigma}_{i-1}(d)\right)\end{array}\right]$ for $i \geq 2$.

Furthermore, if $\left(v^{k}, w^{k}\right)$ is a feasible solution to (18) in the $k^{\text {th }}$ iteration, then the function

$$
F_{C P}(d)=v^{k} d+\sum_{i=1}^{k} w_{i}^{k} F_{i}\left(\sigma_{i}(d)\right)
$$

is a feasible solution to the subadditive dual problem (11).

Wolsey [1981] showed how to construct a dual function optimal to the subadditive dual for a given PILP using the Gomory fractional cutting plane algorithm under the assumption that cuts are generated using a method guaranteed to yield a sequence of LPs with lexicographically increasing solution vectors (this method is needed to guarantee termination of the algorithm in a finite number of steps with either an optimal 
solution or a proof that the original problem is infeasible). In Gomory's procedure, the subadditive function $F_{i}$, generated for iteration $i$, has the following form

$$
F_{i}(d)=\left\lceil\sum_{k=1}^{m} \lambda_{k}^{i-1} d_{k}+\sum_{k=1}^{i-1} \lambda_{m+k}^{i-1} F_{k}(d)\right\rceil \text { where } \lambda^{i-1}=\left(\lambda_{1}^{i-1}, \ldots, \lambda_{m+i-1}^{i-1}\right) \geq 0 .
$$

Assuming that $b \in \Omega, z(b)>-\infty$, and that the algorithm terminates after $k$ iterations, the function $F_{G}$ defined by

$$
F_{G}(d)=v^{k} d+\sum_{i=1}^{k} w_{i}^{k} F_{i}(d)
$$

is optimal to the subadditive dual problem (11).

In practice, it is generally not computationally feasible to determine a subadditive representation for each cut added to the LP relaxation. An alternative approach that is feasible for some classes of valid inequalities is simply to track the dependency of each cut on the original right-hand side in some other way. As an example of this, Schrage and Wolsey [1985] showed how to construct a function tracking dependency on the right-hand side for cover inequalities by expressing the right-hand side of a cut of this type as an explicit function of the right-hand side of the original knapsack constraint.

Corrected Linear Dual Functions. A natural way in which to account for the fact that linear functions are not sufficient to yield strong dual functions in the case of MILPs is to consider dual functions that consist of a linear term (as in the LP case) and a correction term accounting for the duality gap. One way to construct such a function is to consider the well-known group relaxation. Let $B$ be the index set of the columns of a dual feasible basis for the LP relaxation of a PILP and denote by $N \backslash B$ the index set of the remaining columns. Consider the function $F_{B}$ defined as

$$
\begin{aligned}
F_{B}(d)=\min & c_{B} x_{B}+c_{N \backslash B} x_{N \backslash B} \\
\text { s.t } & A_{B} x_{B}+A_{N \backslash B} x_{N \backslash B}=d \\
& x_{B} \in \mathbb{Z}^{m}, x_{N \backslash B} \in \mathbb{Z}_{+}^{n-m} .
\end{aligned}
$$

Substituting $x_{B}=A_{B}^{-1} d-A_{B}^{-1} A_{N \backslash B} x_{N \backslash B}$ in the objective function, we obtain the group relaxation (Gomory [1969])

$$
\begin{aligned}
F_{B}(d)=c_{B} A_{B}^{-1} d-\max & \bar{c}_{N \backslash B} x_{N \backslash B} \\
& A_{B} x_{B}+A_{N \backslash B} x_{N \backslash B}=d \\
& x_{B} \in \mathbb{Z}^{m}, x_{N \backslash B} \in \mathbb{Z}_{+}^{n-m},
\end{aligned}
$$

where $\bar{c}_{N \backslash B}=\left(c_{B} A_{B}^{-1} A_{N \backslash B}-c_{N \backslash B}\right)$. Here, dual feasibility of the basis $A_{B}$ is required to ensure that $\bar{c}_{N \backslash B} \leq 0$.

Note that $F_{B}$ is subadditive and hence feasible to the subadditive dual (11). Observe that $F_{B}(b)=z(b)$ when there exists an optimal solution to (24) with $x_{B} \geq 0$.

Another way to construct an optimal solution to the subadditive dual using a linear function with a correction term is given by Klabjan [2002]. 
Theorem 6 (Klabjan [2002]) For a PILP in the form (1), and a given vector $v \in \mathbb{R}^{m}$, define the function $F_{v}$ as

$$
F_{v}(d)=v d-\max \left\{\left(v A_{\mathcal{D}_{v}}-c_{\mathcal{D}_{v}}\right) x \mid A_{\mathcal{D}_{v}} x \leq d, x \in \mathbb{Z}_{+}^{\left|\mathcal{D}_{v}\right|}\right\},
$$

where $\mathcal{D}_{v}=\left\{j \in I: v a^{j}>c_{j}\right\}$. Then, $F_{v}$ is a feasible solution to the subadditive dual problem (11) and furthermore, if $b \in \Omega$ and $z(b)>-\infty$, there exists $a v \in \mathbb{R}^{m}$ such that $F_{v}(b)=z(b)$.

Klabjan [2002] also introduced an algorithm that finds the optimal dual function utilizing a subadditive approach from (Burdet and Johnson [1977]) together with a row generation approach that requires the enumeration of feasible solutions.

Lagrangian Relaxation. A mathematical program obtained by relaxing and subsequently penalizing the violation of a subset of the original constraints, called the complicating constraints, is a Lagrangian relaxation (Fisher [1981]). Suppose for a given $d \in \mathbb{R}^{m}$ that the inequalities defined by matrix $A$ and right-hand side $d$ are partitioned into two subsets defined by matrices $A^{1}$ and $A^{2}$ and right-hand sides $d^{1}$ and $d^{2}$. Furthermore, let $\mathcal{S}_{L D}\left(d^{1}\right)=\left\{x \in \mathbb{Z}_{+}^{r} \times \mathbb{R}_{+}^{n-r}: A^{1} x=d^{1}\right\}$. Then, for a given penalty multiplier $v \in \mathbb{R}^{m-l}$, the corresponding Lagrangian relaxation can be formulated as

$$
L(d, v)=\min _{x \in \mathcal{S}_{L D}\left(d^{1}\right)} c x+v\left(d^{2}-A^{2} x\right) .
$$

Assuming $z(0)=0$ and that $x^{*}(d)$ is an optimal solution to the original MILP with right-hand side $d$, we have $L(d, v) \leq c x^{*}(d)+v\left(d^{2}-A^{2} x^{*}(d)\right)=c x^{*}(d)=z(d) \forall v \in \mathbb{R}^{m-l}$. Thus, the Lagrangian function defined by

$$
L_{D}(d)=\max \{L(d, v): v \in V\}
$$

with $V \equiv \mathbb{R}^{m-l}$, is a feasible dual function in the sense that $L_{D}(d) \leq z(d) \forall d \in \Omega$. Observe that for a given $d \in \Omega, L(d, v)$ is a concave, piecewise-polyhedral function.

$L_{D}$ above is a weak dual function in general, but Blair and Jeroslow [1979] showed that it can be made strong for PILP problems by introducing a quadratic term.

Theorem 7 (Blair and Jeroslow [1979]) For a PILP in the form (1), denote the quadratic Lagrangian dual function as

$$
L_{D}(d, v)=\max _{\rho \in \mathbb{R}_{+}} L(d, v, \rho)
$$

with

$$
L(d, v, \rho)=\min _{x \in \mathbb{Z}_{+}^{n}}\left\{(c-v A) x+\rho \sum_{i=1}^{m}\left(A_{i} x-d_{i}\right)^{2}+v d\right\},
$$

where $v \in \mathbb{R}^{m}, \rho \in \mathbb{R}_{+}$and $A_{i}$ is the $i^{\text {th }}$ row of $A$. Then for a given $v \in \mathbb{R}^{m}, L_{D}(d, v) \leq z(d) \forall d \in \Omega$. Furthermore, if $b \in \Omega$ and $z(b)>-\infty$, then for any $v \in \mathbb{R}^{m}, L_{D}(b, v)=z(b)$. 
Branch and Bound. Let us assume now that the MILP (1) has a finite optimum and has been solved to optimality by a branch-and-bound algorithm. Let $T$ be the set of leaf nodes of the resulting search tree. To obtain a bound for the node $t \in T$, we solve the LP relaxation of the following problem

$$
\begin{aligned}
z^{t}(b)=\min \quad c x & \\
\text { s.t. } \quad A x & =b \\
x & \geq l^{t} \\
-x & \geq-u^{t} \\
x & \in \mathbb{Z}^{r} \times \mathbb{R}^{n-r},
\end{aligned}
$$

where $u^{t}, l^{t} \in \mathbb{Z}_{+}^{n}$ are the branching bounds applied only to the integer variables.

For each feasibly pruned node $t \in T$, let $\left(v^{t}, \underline{v}^{t}, \bar{v}^{t}\right)$ be the corresponding solution to the dual of the LP relaxation used to obtain the bound that allowed the pruning of node $t$. Note that such a solution is always available if the LP relaxations are solved using a dual simplex algorithm. For each infeasibly pruned node $t \in T$, let $\left(v^{t}, \underline{v}^{t}, \bar{v}^{t}\right)$ be a corresponding dual feasible solution with $v^{t} b+\underline{v}^{t} l^{t}-\bar{v}^{t} u^{t} \geq z(b)$ that can be obtained from the dual solution of the parent of node $t$ and a dual ray that makes the dual problem unbounded.

Theorem 8 (Wolsey [1981]) If $b \in \Omega$ and $z(b)>-\infty$, then the function

$$
F_{B C}(d)=\min _{t \in T}\left\{v^{t} d+\underline{v}^{t} l^{t}-\bar{v}^{t} u^{t}\right\}
$$

is an optimal solution to the dual (5).

Note that it is also possible to consider the dual information revealed by the intermediate tree nodes. Schrage and Wolsey [1985] give a recursive algorithm to evaluate a dual function that approximates the value function better by considering the relations between intermediate nodes and their offsprings.

Generating Functions. Lasserre [2005a,b] introduced a different method for constructing the value function of PILPs that utilizes generating functions. This methodology does not fit well into a traditional duality framework, but nevertheless gives some perspective about the role of basic feasible solutions of the LP relaxation in determining the optimal solution of a PILP.

Theorem 9 (Lasserre [2009]) For a PILP in the form (1) with $A \in \mathbb{Z}^{m \times n}$, define

$$
z(d, c)=\min _{x \in \mathcal{S}(d)} c x
$$

and let the corresponding summation function be

$$
\hat{z}(d, c)=\sum_{x \in \mathcal{S}(d)} e^{c x} \forall d \in \mathbb{Z}^{m} .
$$

Then the relationship between $z$ and $\hat{z}$ is

$$
e^{z(d, c)}=\lim _{q \rightarrow-\infty} \hat{z}(d, q c)^{1 / q} \text { or equivalently, } \quad z(d, c)=\lim _{q \rightarrow-\infty} \frac{1}{q} \ln \hat{z}(d, q c) .
$$


A closed form representation of $\hat{z}$ can be obtained by solving the two sided $\mathbb{Z}-$ transform $\hat{F}: \mathbb{C}^{m} \rightarrow \mathbb{C}$ defined by

$$
\hat{F}(s, c)=\sum_{d \in \mathbb{Z}^{m}} s^{-d} \hat{z}(d, c)=\prod_{j=1}^{n} \frac{1}{1-e^{c_{j}} s^{-a^{j}}},
$$

with $s^{d}=s_{1}^{d_{1}} \ldots s_{m}^{d_{m}}$ for $d \in \mathbb{Z}^{m}$ and where the last equality is obtained by applying Barvinok [1993]'s short form equation for summation problems over the domain of integral points. This equality is well-defined if $\left|s^{a^{j}}\right|>e^{c_{j}}, j=1, \ldots, n$ and the function $\hat{z}$ is then obtained by solving the inverse problem

$$
\hat{z}(d, c)=\frac{1}{(2 i \pi)^{m}} \int_{\left|s_{1}\right|=\gamma_{1}} \cdots \int_{\left|s_{m}\right|=\gamma_{m}} \hat{F}(s, c) s^{d-\mathbf{1}^{m}} d s,
$$

where $\gamma$ is a vector satisfying $\gamma^{a^{j}}>e^{c_{j}} j=1, \ldots, n$ and $\mathbf{1}^{m}=(1, \ldots, 1) \in \mathbb{R}^{m}$.

Although it is possible to solve (36) directly by Cauchy residue techniques, the complex poles make it difficult. One alternative is to apply Brion and Vergne's (see Brion and Vergne [1997], Lasserre [2009] for details) lattice points counting formula in a polyhedron to get the reduced form, which, for each $d \in \mathbb{R}^{m}$, is composed of the optimal solution value of the LP relaxation and a correction term. The correction term is the minimum of the sum of the reduced costs of certain nonbasic variables over all basic feasible solutions, obtained by the degree sum of certain real-valued univariate polynomials. Another approach using generating functions is to apply Barvinok [1994]'s algorithm for counting lattice points in a polyhedron of fixed dimension to a specially constructed polyhedron that includes for any right-hand side the corresponding minimal test set (see Loera et al. [2004a,b] for details).

\section{Conclusion}

Considering the fact that computing the value function for just a single right-hand side is an NP-hard optimization problem in general, computing the value function must necessarily be extremely difficult. The theory of duality, in turn, enables us to derive tractable methods for generating dual functions that approximate the value function and are at the core of a variety of MILP optimization algorithms including sensitivity analysis, warm starting, stochastic and multilevel programming algorithms.

It has so far been difficult to replicate for integer programming the functional and efficient implementations of dual methods we have become so accustomed to in the linear programming case. However, with recent advances in computing, an increased attention is being devoted to integer programming duality in the hope that new computational tools can contribute to more successful implementations.

\section{References}

A. Bachem and R. Schrader. Minimal equalities and subadditive duality. Siam J. on Control and Optimization, 18(4):437-443, 1980. 
A.I. Barvinok. Computing the volume, counting integral points, and exponential sums. Discrete Comput. Geom., 10:1-13, 1993.

A.I. Barvinok. Polynomial time algorithm for counting integral points in polyhedra when the dimension is fixed. Math of Operations Research, 19:769-779, 1994.

C.E. Blair. A closed-form representation of mixed-integer program value functions. Mathematical Programming, 71:127-136, 1995.

C.E. Blair and R.G. Jeroslow. The value function of a mixed integer program: I. Discrete Mathematics, 19: 121-138, 1977.

C.E. Blair and R.G. Jeroslow. The value function of a mixed integer program: II. Discrete Mathematics, 25: 7-19, 1979.

C.E. Blair and R.G. Jeroslow. The value function of an integer program. Mathematical Programming, 23: 237-273, 1982.

C.E. Blair and R.G. Jeroslow. Constructive characterization of the value function of a mixed-integer program: I. Discrete Applied Mathematics, 9:217-233, 1984.

M. Brion and M. Vergne. Residue formulae, vector partition functions and lattice points in rational polytopes. Journal of the American Mathematical Society, 10:797-833, 1997.

C. Burdet and E.L. Johnson. A subadditive approach to solve integer programs. Annals of Discreet Mathematics, 1:117-144, 1977.

M. L. Fisher. The lagrangian relaxation method for solving integer programming problems. Management Science, 27:1-17, 1981.

R.E. Gomory. Some polyhedra related to combinatorial problems. Linear Algebra and Its Applications, 2: 451-558, 1969.

R.G. Jeroslow. Cutting plane theory: Algebraic methods. Discrete Mathematics, 23:121-150, 1978.

R.G. Jeroslow. Minimal inequalities. Mathematical Programming, 17:1-15, 1979.

E.L. Johnson. Cyclic groups, cutting planes and shortest paths. in Mathematical Programming, T.C. Hu and S.M. Robinson (eds.) Academic Press, New York, NY, pages 185-211, 1973.

E.L. Johnson. On the group problem for mixed integer programming. Mathematical Programming Study, 2:137-179, 1974.

E.L. Johnson. On the group problem and a subadditive approach to integer programming. Annals of Discreet Mathematics, 5:97-112, 1979. 
D. Klabjan. A new subadditive approach to integer programming: Theory and algorithms. In Proceedings of the Ninth Conference on Integer Programming and Combinatorial Optimization, pages 384-400, Cambridge,MA, 2002.

J.B. Lasserre. Duality and farkas lemma for integer programs. In Optimization : Structure and Applications, E. Hunt and C.E.M. Pearce, eds.,, Applied Optimization Series, Kluwer Academic Publishers, 2009.

J.B. Lasserre. Generating functions and duality for integer programs. Discrete Optimization, 2(1):167-187, 2005a.

J.B. Lasserre. Integer programming, duality and superadditive functions. Contemporary Mathematics, 374: 139-150, 2005b.

J. De Loera, D. Haws, R. Hemmecke, P. Huggins, B. Sturmfels, and R. Yoshida. Short rational functions for toric algebra and applications. J. Symbolic Comp., 38:959-973, 2004a.

J. De Loera, D. Haws, R. Hemmecke, P. Huggins, and R. Yoshida. Three kinds of integer programming algorithms based on barvinok's rational functions. 10th International IPCO Conference. Lecture Notes in Computer Science, 3064:244-255, 2004b.

L. Schrage and L.A. Wolsey. Sensitivity analysis for branch and bound integer programming. Operations Research, 33(5):1008-1023, 1985.

L.A. Wolsey. The b-hull of an integer program. London School of Economics and CORE, 1979.

L.A. Wolsey. Integer programming duality: Price functions and sensitivity analysis. Mathematical Programming, 20:173-195, 1981. 